\title{
Consecutive Vibrational Redistribution and Predissociation Processes in $s$-Tetrazine-Argon Van Der Waals Complexes
}

\author{
J. J. F. RAMAEKERS, L. B. KRIJNEN, H. J. LIPS, J. LANGELAAR \\ and R. P. H. RETTSCHNICK
}

Laboratory for Physical Chemistry, University of Amsterdam, Nieuwe Achtergracht 127, 1018 WS Amsterdam, The Netherlands

$s$-Tetrazine argon complexes $\mathrm{T}-\mathrm{Ar}_{n}(n=1,2)$ are formed in a supersonic expansion of argon seeded with $s$-tetrazine. The expansion was conducted through a nozzle of 50 or $100 \mu \mathrm{m}$ with an argon stagnation pressure between 1 and 1.5 bar. From spectrally resolved measurements it is clear that vibrational redistribution processes as well as vibrational predissociation processes take place after SVL excitation within the complex.

From rise and decay time experiments it can be concluded, that after excitation of the $6 a^{1}$ complex level, the above mentioned processes are consecutive and not parallel. It appears that the out of plane mode $16 a$ couples with the Van der Waals stretching mode. The predissociation rate of the $16 a^{2}$ complex is observed to be $2.3 \times 10^{9} \mathrm{~s}^{-1}$.

\section{INTRODUCTION}

In a supersonic expansion of $s$-tetrazine seeded in argon, Van der Waals complexes are created during the cooling process. The weakly bound complexes are stable in the region after the nozzle and can thus be studied by laser induced excitation and fluorescence spectroscopy. ${ }^{1-3} \mathrm{The} \mathrm{T}^{-\mathrm{Ar}_{n}}(n=1,2)$ complexes as well as the parent molecule tetrazine can be studied in the supersonic jet under identical conditions by selective excitation. A good comparison then is possible with the known SVL spectra of $s$-tetrazine in a vapor at room temperature 
under isolated conditions and in the single collision regime. ${ }^{4,5}$ After SVL excitation of the complexes, resonance emission from the prepared level is observed in combination with vibronic level emission from other modes which is attributed to either complex emission or to emission from the parent molecule. ${ }^{2,3}$ In this paper we will attribute these results to vibrational relaxation and vibrational predissociation processes. The different channels are followed in time by means of fast decay and rise time experiments. The characterization of the fluorescence emission and excitation spectra is available in literature. ${ }^{1,4,5}$ In a vapor at room temperature ( 0.5 torr) no vibrational relaxation processes are observed in the different vibrational levels studied. On the other hand the decay times observed in the excited state for the different single vibronic levels up to $1000 \mathrm{~cm}^{-1}$ change from $<100 \mathrm{ps}$ up to $1.5 \mathrm{~ns}$. It is assumed that this is due to internal conversion processes followed by photochemical decomposition.

\section{SUPERSONIC JET SPECTROSCOPY}

In the supersonic jet experiments with a nozzle diameter of 50 and $100 \mu \mathrm{m}$ and an Ar stagnation pressure of 1.2 bar with $0.03 \% \mathrm{~s}$ tetrazine vibrational relaxation processes in the tetrazine molecule itself are not observed (i.e., $\leqslant 0.5 \%$ ) in a region after the nozzle from 0 to $5 \mathrm{~mm}$. The dimension of the area excited by the synchronously pumped CW dye laser and observed by a high resolution monochromator in all experiments was of the order of $50 \times 50 \mu \mathrm{m}$.

In contrast to the observation with the molecular species we observed efficient vibrational relaxation and vibrational predissociation effects when the Ar Van der Waals complex species were excited under the same conditions. We have assumed ${ }^{3}$ that soft collisions or orbiting collisions are effective to induce vibrational relaxation in the $s$-tetrazine-Ar complex assisted by perturbation of the molecular vibration by the Van der Waals bound Ar atom. Dispersed fluorescence spectra are obtained with narrow band laser excitation $(\Delta \sigma \simeq$ $0.1 \mathrm{~cm}^{-1}$ ) by means of a 1.5 meter J.Y. grating monochromator $(0.24 \mathrm{~nm} / \mathrm{mm})$ at a resolution of $1 \mathrm{~cm}^{-1}$. Fluorescence excitation and emission spectra were measured for the $0^{0}, 16 a^{2}, 6 a^{1}$ and $6 b^{2}$ levels of the molecular and complex species. The fluorescence excitation spectrum which reveals the $0_{0}^{0}$ transition of the T-Ar complex shifted 
$-23 \mathrm{~cm}^{-1}$ with respect to the molecular transition is presented in Ref. 6 . Blue shifted bands at 34 and $38 \mathrm{~cm}^{-1}$, and $43 \mathrm{~cm}^{-1}$ from the $\mathrm{T}$-Ar complex band are attributed to respectively the Van der Waals bending $(2 \times)$ and stretching modes.

Dispersed fluorescence emission spectra of the $\mathrm{T}-\mathrm{Ar}$ complexes were obtained for the single vibronic levels $\overline{0^{0}}, \overline{16 a^{2}}, \overline{6 a^{1}}$ and $\frac{6 b^{2}}{}$. (The complex levels and transitions are indicated by a bar above the molecular level notation.)

The spectra could be well compared with the known resonance emission spectra of the parent molecule. ${ }^{4}$ The emission spectra of the molecular species and the complex species which have to be compared, were run under the same conditions in the cold molecular jet. The results will be discussed. For each transition, the main observed emission bands are represented in Tables I-IV. The full interpretation of the results will be discussed elsewhere. ${ }^{6}$ The main SVL emission bands of $\mathrm{T}-\mathrm{Ar}$ after $\overline{0_{0}^{0}}$ excitation are given in Table $\mathrm{I}$.

The main transitions from the spectra of tetrazine-argon after $\overline{16 a_{0}^{2}}, \overline{6 b_{0}^{2}}$ and $\overline{6 a_{0}^{1}}$ excitation are summarized in Tables II, III and IV respectively. Relative fluorescence intensities are presented in these tables for the appropriate excitation wavelengths. It is worth noticing that the relative fluorescence intensities are strongly dependent on the excitation wavelength within the broad $\left(3 \mathrm{~cm}^{-1}\right.$, FWHM) excitation band of the particular transition within the complex.

A comparison with the resonance emission of the parent molecule shows that several vibrational levels are populated in the complex as a result of intermolecular or intramolecular vibrational relaxation.

TABLE I

Main bands of the $\overline{0^{0}}$ fluorescence spectrum of T-Ar (Excitation: $\overline{0_{0}^{0}}, 18105 \mathrm{~cm}^{-1}$ )

\begin{tabular}{|c|c|c|c|}
\hline \multirow[b]{2}{*}{ Observed frequency } & \multirow[b]{2}{*}{ Relative intensity } & \multicolumn{2}{|r|}{ Assignment } \\
\hline & & $\mathrm{T}$ & $\mathrm{T}-\mathrm{A}_{\mathrm{n}}$ \\
\hline 17370 & 100 & & $6 a_{1}^{0}$ \\
\hline 17338 & 1.2 & & $6 a_{1}^{0}\left(\beta_{y}\right)_{1}^{0}+6 a_{1}^{0}\left(\beta_{z}\right)_{1}^{0}$ \\
\hline 17328 & 1.5 & & $6 a_{1}^{0}\left(\sigma_{x}\right)_{1}^{0}$ \\
\hline 16635 & 63 & & $6 a_{2}^{0}$ \\
\hline 16603 & 0.4 & & $6 a_{2}^{0}\left(\beta_{y}\right)_{1}^{0}+6 a_{2}^{0}\left(\beta_{z}\right)_{1}^{0}$ \\
\hline 16593 & 0.8 & & $6 a_{2}^{0}\left(\sigma_{x}\right)_{1}^{0}$ \\
\hline
\end{tabular}


TABLE II

Part of the emission bands of T-Ar after excitation in the $\overline{16 a_{0}^{2}}$ transition at $18630 \mathrm{~cm}^{-1}$. (It is inevitable that also the molecular transition $16 a_{0}^{2}$ is excited)

\begin{tabular}{cccc}
\hline & & \multicolumn{2}{c}{ Assignment } \\
\cline { 3 - 4 } Observed frequency & Relative intensity & $\mathrm{T}$ & $\mathrm{T}-\mathrm{Ar}$ \\
\hline 17967 & 239 & $16 a_{2}^{2}$ & \\
17960 & 100 & & $16 a_{2}^{2}$ \\
18046 & 111 & $16 a_{1}^{1}$ & $\left(16 a_{1}^{1}\right)$ \\
18128 & 45 & $00_{0}^{0}$ & \\
18263 & 8 & & $16 b_{1}^{1}$ \\
\hline
\end{tabular}

The emission spectra of $6 a^{1} \mathrm{~T}-\mathrm{Ar}$ and $\mathrm{T}-\mathrm{Ar}_{2}$ were published in Ref. 3 . In Figure 1 we present the emission spectrum after $\overline{6 b_{0}^{2}}$ excitation. The results given in Tables III and IV both for $\overline{6 b_{0}^{2}}$ as well as for $6 a_{0}^{1}$ excitation indicate, that as a result of vibrational relaxation within the complex, mainly the $\frac{16 a^{2}}{16 a^{1} 16 b^{1}}$ and $5^{1}$ levels are formed.

Vibrational predissociation, a relaxation process of the complex with ejection of the argon atom, mainly produces tetrazine in the $16 a^{1}$ and $16 b^{1}$ level.

TABLE III

Part of the emission bands of T-Ar after excitation of the $\overline{6 b^{2}}$ level $\left(18889 \mathrm{~cm}^{-1}\right)$

\begin{tabular}{cccc}
\hline & & \multicolumn{2}{c}{ Assignment } \\
\cline { 3 - 4 } Observed frequency & Relative intensity & $\mathrm{T}$ & $\mathrm{T}-\mathrm{Ar}$ \\
\hline 18388 & 32 & $16 b_{1}^{1}$ & $6 b_{0}^{2} 16 b_{2}^{0}$ \\
18280 & 32 & & $16 a_{1}^{1} 16 b_{1}^{1}$ \\
18187 & 30 & $16 a_{1}^{1}$ & $6 b_{0}^{2} 6 a_{1}^{0}$ \\
18157 & 100 & & $16 a_{2}^{2}$ \\
18048 & 77 & & $5_{1}^{1}$ \\
17962 & 40 & & \\
17790 & 10 & &
\end{tabular}


TABLE IV

Part of the emission bands of T-Ar after excitation of the $\overline{6 a^{1}}$ level $\left(18809 \mathrm{~cm}^{-1}\right)$

\begin{tabular}{|c|c|c|c|}
\hline \multirow[b]{2}{*}{ Observed frequency } & \multirow[b]{2}{*}{ Relative intensity } & \multicolumn{2}{|c|}{ Assignment } \\
\hline & & $\mathrm{T}$ & $\mathrm{T}-\mathrm{Ar}$ \\
\hline 18765 & & & $6 a_{0}^{1}\left(\sigma_{x}\right)_{1}^{0}$ \\
\hline 18304 & 14 & & $6 a_{0}^{1} 16 b_{2}^{0}$ \\
\hline 18268 & 4 & & $16 b_{1}^{1}$ \\
\hline 18182 & 13 & & $16 a_{1}^{1} 16 b_{1}^{1}$ \\
\hline 18138 & 16 & & $6 a_{0}^{1} 16 a_{2}^{0}$ \\
\hline 18123 & 2 & & \\
\hline 18073 & 8 & & $6 a_{1}^{1}$ \\
\hline 18048 & 99 & $16 a_{1}^{1}$ & \\
\hline 18008 & 2 & & $6 a_{0}^{1} 4_{1}^{0}$ \\
\hline 17961 & 37 & & $16 a_{2}^{2}$ \\
\hline 17892 & 2 & & \\
\hline 17862 & 1 & & \\
\hline 17855 & 1 & & $6 a_{0}^{1} 1_{1}^{0}$ \\
\hline 17799 & 2 & & \\
\hline 17787 & 7 & & $5_{1}^{1}$ \\
\hline 17585 & 2 & & $6 a_{0}^{1} 16 a_{1}^{0} 16 b_{1}^{0} 6 b_{1}^{0}$ \\
\hline 17565 & 3 & & $6 a_{1}^{1} 16 b_{2}^{0}$ \\
\hline 17528 & 13 & & $6 a_{0}^{1} 6 b_{2}^{0}$ \\
\hline 17446 & 10 & & $16 a_{1}^{1} 16 b_{1}^{1} 6 a_{1}^{0}$ \\
\hline 17404 & 3 & & $6 a_{1}^{1} 16_{2}^{0}$ \\
\hline 17394 & 17 & & $6 a_{0}^{1} 8 a_{1}^{0}$ \\
\hline 17370 & 9 & $16 a_{3}^{1}$ & \\
\hline 17337 & 100 & & $6 a_{2}^{1}$ \\
\hline 17312 & 86 & $16 a_{1}^{1} 6 a_{1}^{0}$ & \\
\hline 17294 & 2 & & $6 a_{2}^{1}\left(\sigma_{x}\right)_{1}^{0}$ \\
\hline 17279 & 6 & & $16 a_{4}^{2}$ \\
\hline 17226 & 26 & & $16 a_{2}^{2} 6 a_{1}^{0}$ \\
\hline 17207 & 3 & & $6 a_{0}^{1} 4_{2}^{0}$ \\
\hline 17142 & 2 & & \\
\hline
\end{tabular}

\section{KINETICS}

In addition to the spectra, we have performed time resolved experiments to analyze the individual kinetics and to deduce whether the different processes are taking place parallelly or are consecutive. In this paper we will analyze the results obtained for the T-Ar complex excited in the $\overline{6 a^{1}}$ and $\overline{16 a^{2}}$ level. 


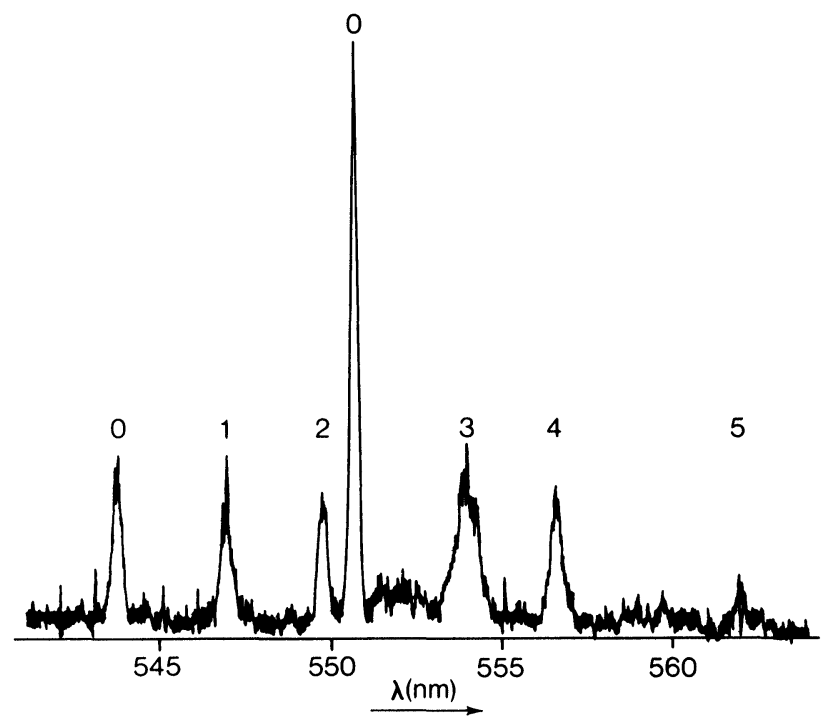

FIGURE 1a Part of the fluorescence emission spectrum of the $s$-tetrazine argon complex after $\overline{6 b_{0}^{2}}$ excitation. $\left(18889 \mathrm{~cm}^{-1}\right)$. Detection bandwidth $=10 \mathrm{~cm}^{-1} .0$ : Resonance emission, 1: $16 b^{1}$ emission, 2: $\overline{16 a^{1} 16 b^{1}}$ emission, 3: $16 a^{1}$ emission, 4: $\overline{16 a^{2}}$ emission, 5: $\overline{5^{1}}$ emission.

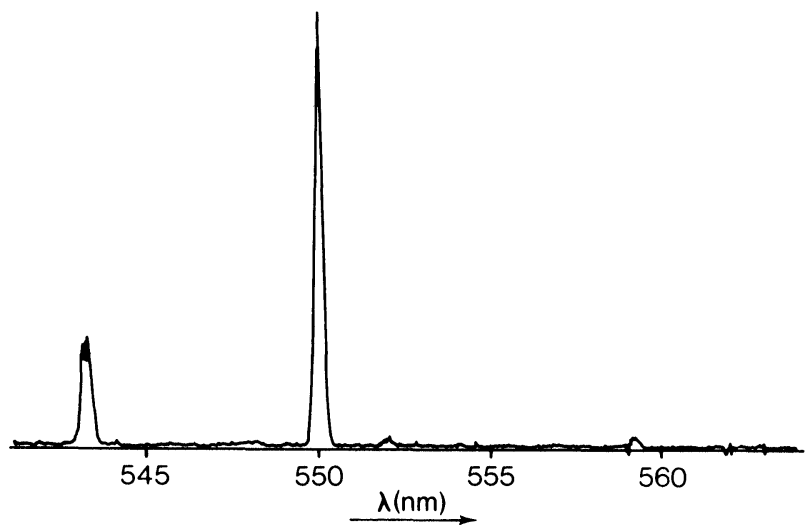

FIGURE 1b Part of the fluorescence emission spectrum of the $s$-tetrazine molecule after $6 b_{0}^{2}$ excitation. $\left(18912 \mathrm{~cm}^{-1}\right)$. Detection bandwidth $=10 \mathrm{~cm}^{-1}$. Note: all emission originates from the prepared $6 b^{2}$ level. 
After excitation in the $\overline{6 a^{1}}$ level the emission spectrum tells us that the vibrational energy mainly flows to the $\overline{16 a^{2}}$ mode of the complex and to the $16 a^{1}$ mode of the molecular species.

The first process

$$
\overline{6 a^{1}} \longrightarrow \overline{16 a^{2}}
$$

must be due to vibrational redistribution; the second process due to vibrational predissociation either from the $6 a^{1}$ level directly i.e.,

$$
\overline{6 a^{1}} \longrightarrow 16 a^{1}+\mathrm{Ar}
$$

or from a consecutive process of process (1) i.e.,

$$
\overline{16 a^{2}} \longrightarrow 16 a^{1}+\mathrm{Ar}
$$

A first indication is obtained from the graphically determined decay times for the three levels, as compared with the decaytimes of $s$ tetrazine in the same vibrational levels (Table V).

In the $6 a^{1}$ level a similar decay time is observed for excitation of the molecule and for the argon complex. This indicates that the processes (1) and (2) do not compete with the main deactivation process, i.e., the photodissociation of $s$-tetrazine into its products $\mathrm{HCN}$ and $\mathrm{N}_{2}$. For the $16 a^{2}$ level we see a drastic reduction of the decay time indicating that the predissociation process $(1 a)$ is an extra channel competing with the photodecomposition of the molecule. A similar reduction in decaytime is observed after direct excitation of the $\overline{16 a^{2}}$ level of the complex. Here the predissociation channel

$$
\overline{16 a^{2}} \longrightarrow 16 a^{1}+\mathrm{Ar}
$$

also reduces the decay time of the $\overline{16 a^{2}}$ level. In order to proof these interpretations we performed risetime experiments for the different levels mentioned in the processes (1) to (3). For this we used

TABLE V

Graphically determined decay times (ps) for several levels

\begin{tabular}{rrrrrr}
\hline \multicolumn{2}{c}{$s$-Tetrazine } & \multicolumn{2}{c}{$\begin{array}{c}\text { T-Ar } \\
\left.\text { (after exc in } \overline{6 a_{0}^{1}}\right)\end{array}$} & $\begin{array}{c}\text { T-Ar } \\
\left(\text { after exc in } \overline{16 a^{2}}\right)\end{array}$ \\
\hline $6 a^{1}$ & 520 & $\overline{6 a^{1}}$ & 520 & & \\
$16 a^{2}$ & 1540 & $\overline{16 a^{2}}$ & $\sim 600$ & $\overline{16 a^{2}}$ & $\sim 800$ \\
$16 a^{1}$ & 1450 & $16 a^{1}$ & 1500 & $16 a^{1}$ & 1400 \\
\hline
\end{tabular}


a fast cross-field photomultiplier with a risetime of $80 \mathrm{ps}$ (10 to $90 \%$ ). Unfortunately the decay time of this detector was not as fast as its rise-time, the cause of which is not understood yet. It will, however, not influence the decay times presented in this paper.

The growth and decay of the prepared $\overline{6 a^{1}}$ level of the T-Ar complex was measured and is represented in Figure 2. The observed growth of this resonance emission equals the risetime of the detector and electronics, because the excitation was performed with a short ( $7 \mathrm{ps}, \mathrm{FWHM}$ ) pulse from the synchronously pumped dye laser. The decay time obtained for the $\overline{6 a^{1}}$ complex level is $520 \mathrm{ps}$, similar to that observed for the molecular species in the $6 a^{1}$ level (Table V).

The experimental growth and decay curve of the formed $\overline{16 a^{2}}$ level is represented in Figure 3 (dotted curve). If we assume, according to process (1), that the $\overline{16 a^{2}}$ level is formed directly from the excited $6 a^{1}$ level, then the growth and decay has to be a convolution of the pulseform of the $\overline{6 a^{1}}$ emission with the decay time of the $\overline{16 a^{2}}$ level. The best fit with the experimental curve is obtained with a decay time of $330 \mathrm{ps}$ (Figure 3, drawn curve). It is worth to mention

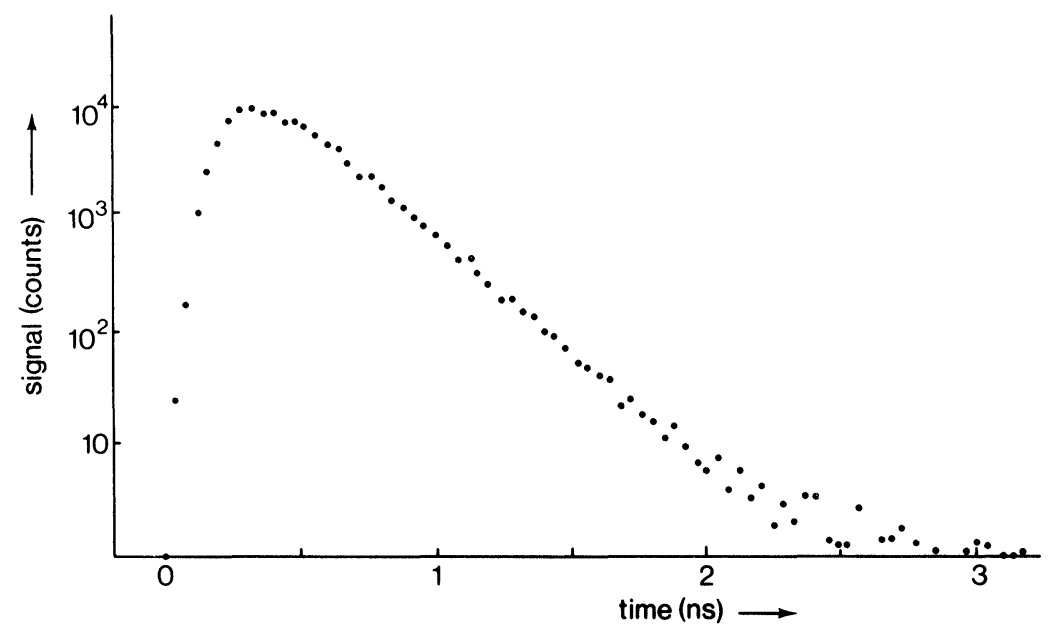

FIGURE 2 Experimental growth and decay curve of the $\overline{6 a^{1}}$ fluorescence emission after excitation within the $\overline{6 a_{0}^{1}}$ band of the $s$-tetrazine-argon Van der Waals complex. Decaytime $\tau\left(\overline{6 a^{1}}\right)=520$ ps. 


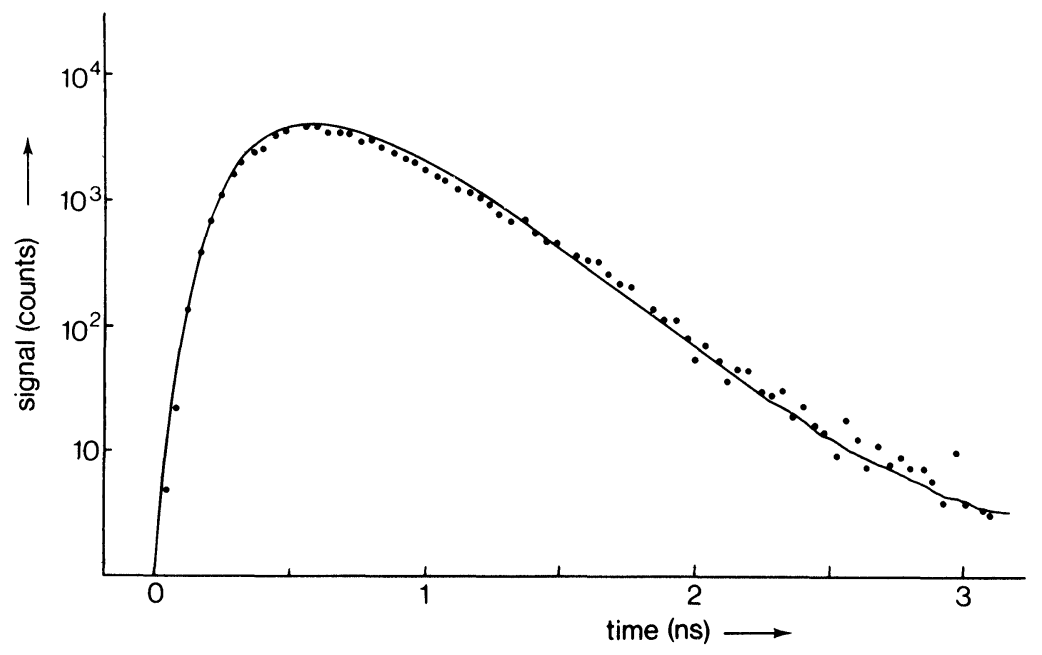

FIGURE 3 Dotted curve: Experimental growth and decay curve of the $\overline{16 a^{2}}$ emission after $\overline{6 a_{0}^{1}}$ excitation. Drawn curve: Best fit computer simulation for this emission using the experimental curve of Figure 2 as input. Process: $\overline{6 a^{1}} \rightarrow \frac{16 a^{2}}{\text {; }}$ Decaytime $\tau\left(\overline{16 a^{2}}\right)=330 \mathrm{ps}$.

here that this decay time is a factor of 2 shorter than that graphically obtained from the logarithmic plot of the experimental curve, not taking into account the growth and decay of the level from which it is formed as is the case for the numbers given in Table V and Ref. 3.

Finally the growth and decay of the $16 a^{1}$ level was measured and compared with the calculated curve as if it was formed either by process $(1 a)$ or by process (2). If the $16 a^{1}$ level is formed according to the predissociation process $(1 a)$ than a convolution of the pulseform of the $\overline{16 a^{2}}$ emission with the appropriate $16 a^{1}$ decay time has to give the best fit between calculated and experimental curve. If on the other hand process (2) is the correct channel for the predissociation process than the convolution has to be carried out with the pulseform of the $6 a^{1}$ emission. The best fit is obtained in agreement with process (1a) (Figure 4). Hence the results indicate that process (2) can be neglected, which brings us to the conclusion that predissociation of the $\mathrm{T}-\mathrm{Ar}$ complex does not take place directly from the prepared $\frac{6 a^{1}}{1}$ level. 


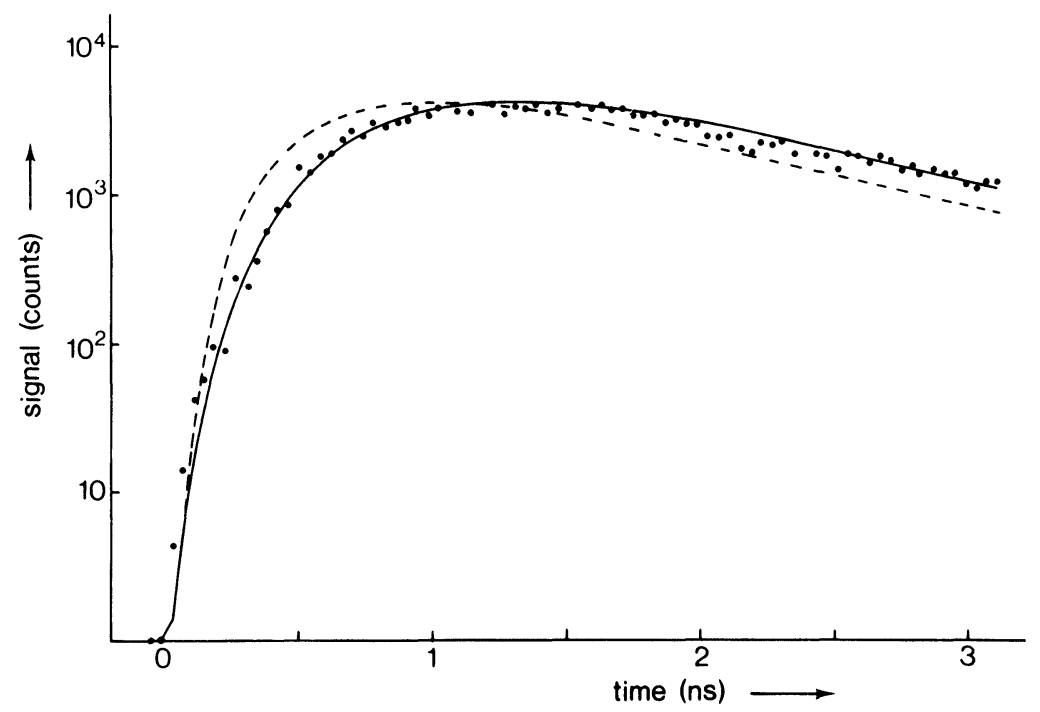

FIGURE 4 Dotted curve: Experimental growth and decay curve of the $16 a^{1}$ emission after $6 a_{0}^{1}$ excitation. Drawn curve: Best fit computer simulation for the $16 a^{1}$ emission using the drawn curve of Figure 3. Process: $16 a^{2} \rightarrow 16 a^{1}$; Decaytime $\tau\left(16 a^{1}\right)=$ 1450 ps. Dashed curve: Computer simulation for $16 a^{1}$ emission using the curve of Figure 2 as input. A good fit cannot be obtained. Process: $\overline{6 a^{1}} \rightarrow 16 a^{1}$; Decaytime $\tau\left(16 a^{1}\right)=1450$ ps.

On the other hand vibrational predissociation is appreciable from the $\overline{16 a^{2}}$ level of the complex. We might conclude that a $s$-tetrazine argon complex prepared in an in-plane vibrational mode $\frac{6 a^{1}}{16}$ first has to redistribute its vibrational energy to an out of plane $\frac{16 a^{2}}{16}$ complex mode before it predissociates to a molecular species in the $16 a^{1}$ level and an argon atom. Hence vibrational relaxation and vibrational predissociation of a Van der Waals complex are consecutive processes and do not take place parallelly from the prepared $6 a^{1}$ level. From the results presented in Figure 3 we calculate a vibrational predissociation rate of $2.3 \times 10^{9} \mathrm{~s}^{-1}$ for the $\frac{16 a^{2}}{16 a^{2}}$ level. A similar result is obtained by kinetic studies of the $\frac{16 a^{2}}{16 a^{2}}$ level after direct excitation of the $\overline{16 a_{0}^{2}}$ band. Clearly, a coupling between the out of plane $16 a$ mode of $s$-tetrazine and the Van der Waals stretching mode $\left(43 \mathrm{~cm}^{-1}\right)$ is also illustrated by the results presented in this paper (see also Ref. 6). 


\section{Acknowledgement}

The authors are indebted to Mr. D. Bebelaar for developing the fast detection equipment. The investigations were supported in part by the Netherlands Foundation for Chemical Research (SON) with financial aid from the Netherlands Organization for Advancement of Pure Research (ZWO).

\section{References}

1. R. E. Smalley, L. Wharton, D. H. Levy and D. W. Chandler, J. Chem. Phys. 68, 2487 (1978).

2. J. E. Kenny, D. V. Brumbaugh and D. H. Levy, J. Chem. Phys. 71, 4757 (1979).

3. J. J. F. Ramaekers, J. Langelaar and R. P. H. Rettschnick, Picosecond Phenomena III, eds. K. B. Eisenthal, R. M. Hochstrasser, W. Kaiser and A. Laubereau (Springer Verlag, 1982) pp. 264-268.

4. M. W. Leeuw, Thesis, University of Amsterdam 1981; M. W. Leeuw, J. Langelaar and R. P. H. Rettschnick, Proc. of the UPS 80, Reinhardsbrunn DDR, pp. 220-224 (1980).

5. D. V. Brumbaugh and K. K. Innes, Chem. Phys. 59, 413 (1981).

6. J. J. F. Ramaekers, H. K. van Dijk, J. Langelaar and R. P. H. Rettschnick, Faraday Disc. Chem. Soc. (in press). 